\title{
Leitlinien zu den historischen Avantgarden Lateinamerikas
}

Es gibt gute Gründe dafür, erst mit den hispanoamerikanischen „Modernistas“ die Entstehung eines Bewusstseins für ein gemeinsames Schreiben in der spanischsprachigen Welt der Amerikas anzusetzen. Gegen Ende des 19. Jahrhunderts war durch vielfältige Reisen und persönliche Begegnungen die wechselseitige Kenntnis zwischen den Autorinnen und Autoren der spanischsprachigen Welt gestärkt und durch die gehäufte Herausgabe literarischer Zeitschriften kontinentalen (oder subkontinentalen) Zuschnitts intensiviert worden, so dass der Austausch zwischen den verschiedenen Teilregionen der lateinamerikanischen Welt sich rasch entfalten konnte.

Mit den historischen Avantgarden in Lateinamerika setzt sich dieser Prozess beschleunigt fort. Die Verbindungen zwischen ästhetischer und politischer Avantgarde lassen sich nicht zuletzt am Beispiel verschiedener Zeitschriften wie der Revista Amauta in Peru oder der Revista de Avance in Kuba aufzeigen. Sie machen deutlich, dass grundlegende Diskussionen im politischen wie im ästhetischen Feld längst nicht mehr nur lokal, sondern gesamtlateinamerikanisch geführt wurden, schrieben doch verschiedenste Intellektuelle nun in den überregional konzipierten (wenn auch natürlich lokal verorteten) Zeitschriften der gesamten lateinamerikanischen Welt von Mexiko bis Chile.

Diese Entwicklungen konsolidierten sich im Übrigen auch im weiteren Verlauf des 20. Jahrhunderts oder nahmen an Bedeutung noch zu: Politische und ästhetisch-literarische Avantgarden betrieben einen intellektuellen Schulterschluss, der ebenso für das literarische Teilfeld wie auch vor allem für das politische Feld fruchtbare Folgen zeitigte. Dies lässt sich ebenso für die kubanischen Zeitschriften der dreißiger, vierziger und fünfziger Jahre (wie etwa Verbum, Espuela de Plata oder Orígenes wie auch für Zeitschriften des südamerikanischen Subkontinents zeigen, bei denen der Revista Sur - wie wir noch sehen werden - in Argentinien eine besondere Bedeutung gerade auch hinsichtlich der europäisch-lateinamerikanischen Beziehungen zukam.

In der Folge möchte ich auf einige herausragende Vertreter der historischen Avantgarden in Hispanoamerika, nämlich Vicente Huidobro und César Vallejo, aber auch auf den Spanier Ramón Gómez de la Serna näher eingehen. Danach werde ich eine Gruppe von Lyrikerinnen behandeln, die freilich nicht mehr unmittelbar den Avantgarden zuzurechnen sind, sondern uns auf spezifische Bedingungen und Ausdrucksformen weiblichen Schreibens aufmerksam machen sollen. Doch zuvor will ich noch einige generelle Leitlinien - in insgesamt acht Thesen zusammengefasst - in Hinblick auf die Vorlesung formulieren, um diese 
Überlegungen für unsere Beschäftigung mit den Avantgarden fruchtbar machen zu können

Erstens setzen die historischen Avantgarden in Hispanoamerika gegenüber jenen in Europa mit einer gewissen Zeitverzögerung ein, obwohl die Übermittlungen der ersten Manifeste binnen weniger Wochen bewerkstelligt wurden und die neuen europäischen Entwicklungen in Lateinamerika rasch in den großen Städten des Subkontinents bekannt geworden waren. Betrachten wir allerdings die ersten Manifeste des Chilenen Vicente Huidobro, dann relativiert sich diese scheinbare Verzögerung gewaltig! Die historischen Avantgarden prägen sich in den unterschiedlichen literarischen Teilregionen wie etwa der Karibik, Brasilien, den Andenländern, Mexiko und Mittelamerika sowie dem Cono Sur sehr unterschiedlich aus und bilden eine überaus charakteristische Heterogenität, die es sehr wohl erlaubt, mit Blick auf Lateinamerika von unterschiedlichen Literaturen zu sprechen. ${ }^{1}$

Zugleich ist jedoch, wie oben ausgeführt, die interne Vernetzung zwischen den einzelnen Teilregionen gerade durch die Wirksamkeit bestimmter Periodika und Zeitschriften überregionalen Zuschnitts gewährleistet. Wir werden die Chronologie dieser an verschiedensten Orten und Regionen in Lateinamerika auftauchenden Avantgarden am Beispiel des Chilenen Vicente Huidobro überprüfen, ohne dabei zu vergessen, dass es kaum weniger frühe starke avantgardistische Bewegungen nicht nur in Chile, sondern auch in Peru, Argentinien, Uruguay, Brasilien, Kolumbien, Venezuela, Nikaragua, Mexiko, Kuba sowie - mit relativ großer Verspätung - auch in der Dominikanischen Republik gab. Die jeweiligen Einzelentwicklungen können im Rahmen dieser Vorlesung selbstverständlich nicht vorgestellt werden, sollten uns aber bei unseren Analysen bewusst bleiben.

Zweitens hat die unbestreitbare Tatsache, dass man diese zum Teil gegenläufigen literarischen Phänomene unter dem in den Plural gestellten Etikett der ,Avantgarden' subsumieren kann, wesentlich mit der seit den hispanoamerikanischen Modernisten beobachtbaren Erscheinung direkter kontinentaler Verbindungen und eines Zusammengehörigkeitsgefühls zu tun. Letzteres lässt sich unter anderem auch mit einer intensiven Kommunikation nicht allein vermittels

1 Vgl. hierzu die Arbeiten von Klaus Müller-Bergh und Gilberto Mendonça Teles: (Hg.): Vanguardia latinoamericana. Historia, crítica y documentos. Tomo I: México y América Central. Tomo II: Caribe, Antillas Mayores y Menores. Frankfurt am Main - Madrid: Vervuert - Iberoamericana 2000-2002; dies. (Hg.): Tomo III: Sudamérica. Area Andina Norte: Colombia - Venezuela. Madrid - Frankfurt am Main: Iberoamericana - Vervuert 2004; dies. (Hg.): Tomo IV: Sudamérica: Area Andina Centro: Ecuador - Peru - Bolivia. Madrid - Frankfurt am Main: Iberoamericana Vervuert 2005; dies. (Hg.): Tomo V: Sudamérica. Chile y países del Plata: Argentina - Paraguay Uruguay. Frankfurt am Main - Madrid: Vervuert - Iberoamericana 2009. 
literarischer Zeitschriften, sondern auch mit längeren persönlichen Aufenthalten, aber auch Exilerfahrungen in jeweils anderen Ländern Lateinamerikas in Verbindung bringen. Es ist diese Gemengelage von literarischen Zeitschriften und persönlichen Kontakten, welche für einen gemeinsamen Problemhorizont und kulturellen Austausch zwischen den Avantgarden der verschiedenen literarischen Regionen verantwortlich zeichnet. Dabei ist die ehemalige Frontstellung gegenüber der einstigen Kolonialmacht Spanien, die ihren letzten Kolonialkrieg im spanisch-kubanisch-US-amerikanischen Konflikt und Waffengang 1898 verlor, längst in den Hintergrund gerückt und macht einer zunehmenden Abwehrhaltung gegenüber den weiter vordringenden Vereinigten Staaten und ihrer Vielzahl an Interventionen sowie ihrer Kanonenboot-Politik Platz.

Zugleich zeigen aber auch einige Lebensläufe führender hispanoamerikanischer Avantgardisten, dass die Außensicht ebenfalls von größter Wichtigkeit für die historischen Avantgarden wurde. Hielten sich doch Dichter und Schriftsteller wie der Chilene Vicente Huidobro, der Peruaner César Vallejo, der Mexikaner Alfonso Reyes, der Kubaner Alejo Carpentier, der Guatemalteke Miguel Angel Asturias, der Argentinier Oliverio Girondo und viele andere mehr in Paris auf, wo es nicht nur ausgedehnte lateinamerikanische Zirkel, sondern auch Zeitschriften gab, in welchen diese Autoren publizieren konnten. Die Rolle der "ville-lumière“ für die lateinamerikanischen Avantgarden ist folglich nicht zu unterschätzen, bildete Paris doch stets einen Brückenkopf, innerhalb dessen intensive Vermittlungsprozesse zwischen den europäischen wie den lateinamerikanischen Avantgarden stattfinden konnten. Von herausragender Bedeutung scheint mir dabei die Erfahrung zu sein, welche die Begegnungen und Erlebnisse des Exils der sechziger und siebziger Jahre in Europa vorwegnimmt und zugleich vorbereitet: Das Bewusstsein, dass nicht mehr nur die eigene Nation und deren Entwicklung, sondern aus der Distanz diejenige der Literaturen der gesamten spanisch- und auch der portugiesisch-sprachigen Welt Amerikas sichtbar und intensiv rezipiert wurde. Das lateinamerikanische Exil dieser Jahre in Europa verstärkte somit all jene Entwicklungen, welche dazu führten, dass die lateinamerikanischen Länder immer weniger einem verstreuten Archipel glichen, dessen einzelne Inseln durch immense Festlandsflächen voneinander getrennt waren. Gleichwohl ist eine archipelische Situation der lateinamerikanischen Welt bis heute unbestreitbar und auch nachprüfbar. ${ }^{2}$

Drittens lässt sich innerhalb bestimmter literarischer Teilregionen eine prononciertere Verknüpfung von literarischer und politischer Avantgarde beob-

2 Vgl. Ribeiro, Darcy: Gibt es Lateinamerika? In (ders.): Unterentwicklung, Kultur und Zivilisation. Ungewöhnliche Versuche. Frankfurt am Main: Suhrkamp 1980, S. 315-328. 
achten, die eng verbunden ist mit jenen politisch-ideologischen Frontstellungen, welche das gesamte 20. Jahrhundert prägen sollten. Dabei ist auffällig, dass diese politische Dimension avantgardistischen Schreibens und avantgardistischen Selbstverständnisses gerade in jenen literarischen Regionen stark ist, in denen es größere ethnische Gruppen wie etwa eine indigene oder eine schwarze Bevölkerung gibt. Diese Gruppen nahmen und nehmen zumeist weiterhin innerhalb der Gesamtgesellschaft - oder genauer: innerhalb des politischen Konstrukts und Gebildes des Nation-Staats (des „estado-nación“) - eine relativ isolierte und marginalisierte Position ein.

In so verschiedenen Ländern wie Mexiko, Kuba oder Peru spielte für die politisch-ästhetischen Avantgarden jeweils die Integration dieser Bevölkerungsgruppen in die Gesamtgesellschaft eine überragende Rolle. Ganz so, wie Alfonso Reyes in Ifigenia cruel die indigene Position zu integrieren und mit dem Schicksal einer gesamten Gemeinschaft zu versöhnen suchte, so entwickelte in Kuba beispielsweise der junge Alejo Carpentier in seinem ¡Ecué-Yamba-Ô! Narrationen, die ebenso drastisch auf das Schicksal der Schwarzen aufmerksam machten wie Nicolás Guillén in vielen seiner avantgardistischen Klanggedichte wie etwa Sóngoro cosongo. ${ }^{3}$

Viertens ist insbesondere in jenen Regionen, die über einen hohen Anteil an marginalisierter indigener oder schwarzer Bevölkerung verfügen, die politische Avantgarde mit der Herausforderung einer Identitätsfindung und dabei vor allem der Problematik einer angemessenen sozialen und politischen, vor allem aber auch kulturellen Partizipation dieser Bevölkerungsgruppen verbunden und beschäftigt. Die vorgeschlagenen Lösungsansätze reichen von „mestizaje“ und „transculturación“ bis hin zu einer Umkehrung der kulturellen Dominanzen im Kontext einer grundlegenden Veränderung des Bildungssystems in den Ländern Lateinamerikas. ${ }^{4}$ Gerade die Theorie der Transkulturation des kubanischen Anthropologen Fernando Ortiz ist aufs Engste mit avantgardistischen Vorstellungen und soziopolitischen Bewegungen verknüpft und kündet von der Symbiose literarisch-wissenschaftlicher Studien und politisch-avantgardistischer Überzeugungen.

3 Vgl. Ette, Ottmar: Nicolás Guillén: Stimme der Lyrik - Lyrik der Stimme. In: Romanistische Zeitschrift für Literaturgeschichte / Cahiers d'Histoire des Littératures Romanes (Heidelberg) XXVII, 1-2 (2003), S. 209-238.

4 Vgl. zu diesen Theoriekonzepten Ette, Ottmar: Transatlantische Transplantationen: Von Pfropfung und „mestizaje“ zum transarchipelischen Zusammenleben in den Amerikas. In (ders. / Wirth, Uwe, Hg.): Kulturwissenschaftliche Konzepte der Transplantation. Unter Mitarbeit von Carolin Haupt. Berlin - Boston: Walter de Gruyter 2019, S. 29-65. 
Die historischen Avantgarden in Lateinamerika erweisen sich mithin keineswegs als mehr oder minder esoterische, artifizielle Gebilde, sondern setzen die von den Modernisten begonnene Diskussion um die Identität Lateinamerikas nun unter anderen, noch dringlicher gewordenen politisch-sozialen Vorzeichen fort. Die fast obsessiv verfolgte Problematik der Identitätsbildung und -suche scheint mir hierbei ein distinktives Merkmal der hispanoamerikanischen Avantgarden zu sein, wie kritisch man auch immer gegenüber dem problembehafteten und gesellschaftlich gefährlichen Begriff der ,Identität‘ sein muss. Zudem macht diese Situation darauf aufmerksam, dass neben die von den historischen Avantgarden bevorzugten Gattungen Lyrik und Theater nun auch verstärkt der Essay tritt, für den sich leicht Beispiele aus Peru, Argentinien, Chile oder Mexiko beibringen ließen. Dazu treten weitere Formen politisch-literarischer Prosa wie etwa die Manifeste der Studentenbewegung, eine durchaus verbreitete Gattung, auf die wir im spezifisch literarischen Bereich zurückkommen werden.

Fünftens unterscheiden sich die historischen Avantgarden in Hispanoamerika hinsichtlich der Problematik des Bruchs, wie sie Peter Bürger in seiner Theorie der Avantgarde postuliert hat, sehr deutlich von den europäischen Spielarten avantgardistischen Schreibens und künstlerischer Gestaltung. Dies verwundert uns nicht, hatten wir doch festgestellt, dass die allgemeinen Theoriebildungen Bürgers (und vieler anderer) ausschließlich an europäischen Modellen orientiert waren. Obwohl sie selbst in Europa kaum einmal über den französisch-, italienisch-, englisch- oder deutschsprachigen Bereich hinausgriffen und dabei nur in den seltensten Fällen den spannenden Bereich spanischer, russischer oder ungarischer Avantgardebewegungen miteinbezogen, glaubten sie dennoch, die Ergebnisse ihrer theoretischen Forschungen ungeprüft verallgemeinern $\mathrm{zu}$ können. Eine Theorie der Avantgarde im Sinne Peter Bürgers bleibt eine literaturwissenschaftliche Chimäre.

In den verschiedenen Ländern Lateinamerikas lässt sich insgesamt eine große Kontinuität der kulturellen Traditionen in allen literarischen Teilregionen beobachten, während sich die Problematik des Bruchs in verschiedener Weise vor allem auf den politisch-ideologischen Bereich, die gesellschaftlich-kulturelle Zusammenstellung und die Konvivenz zwischen den verschiedenen ethnischen Gruppen sowie auf die Institutionalisierung von Literatur und Kunst bezieht. Den historischen Avantgarden ist der ausgeprägte Wunsch gemeinsam, größere Teile der Bevölkerung an Kultur im Allgemeinen und Literatur im Besonderen partizipieren zu lassen. Diese kulturkritische und zum Teil gegen die Institutionen Literatur und Kunst orientierte Stoßrichtung ist freilich unterschiedlich stark ausgeprägt. Sie steht zudem angesichts der soziopolitischen und kulturellen Prämissen trotz steigender Alphabetisierungsraten bei insgesamt deutlich kleineren Leserschichten in Lateinamerika innerhalb anderer gesellschaftlicher Kontexte 
und ist logischerweise anders $\mathrm{zu}$ bewerten. Die literarische Sozialisierung dieser Autoren erfolgte zumeist noch im hispanoamerikanischen Modernismo und dessen unterschiedlichen Spielarten wie etwa dem auf Rodós Ariel zurückgehenden „Arielismo“. Es fällt angesichts dieser Kontinuitäten - wie wir sahen - nicht immer leicht, eine klare Trennlinie zwischen Modernismo und „Vanguardia“ zu ziehen, insofern eine scharfe Separation, wie sie uns vor allem aus dem Umgang mit den europäischen Literaturen vertraut ist, fehl am Platz ist. Bei derartigen Versuchen handelt es sich zumeist um eine unreflektierte Übertragung europäischer Kategorisierungen.

Sechstens äußert sich das Ziel einer stärkeren politischen wie kulturellen Partizipation breiter Bevölkerungsschichten sehr unterschiedlich und strebt entweder nach einer stärkeren Distribution von Literatur, wie sie etwa die frühe Lyrik Oliverio Girondos mit spezifischen Lektürevorgaben in seinen Veinte poemas para ser leídos en el tranvía (Zwanzig Gedichte, in der Straßenbahn zu lesen) anstrebte, oder kommt vor allem in dem Versuch zum Ausdruck, durch den Rückgriff auf volkskulturelle Ausdrucksformen, welche einen wichtigen kulturellen Pol in Lateinamerika bilden, breite Bevölkerungskreise in eine zumeist national definierte Kultur zu integrieren.

Dies ist jeweils in Zusammenhang mit den Bemühungen zu sehen, den Pol der europäisch-abendländischen Kulturen und Literaturen mit den verschiedenen indigenen Kulturen oder mit den nicht weniger komplexen Kulturen afrikanischen Ursprungs zu verknüpfen. Daraus entstehen avantgardistische Kunst- und Literaturformen, welche - wie etwa bei Alfonso Reyes - auf eine symbiotischere oder - wie etwa beim frühen Carpentier - auf eine etwas radikalere Art und Weise die angestrebte Konvivenz der Kulturen ins Auge fassen. Dies ist zweifellos ein Spezifikum der historischen Avantgarden in Lateinamerika, auch wenn das in Europa erwachte Interesse an den sogenannten ,primitiven' Kulturen - vorzugsweise in Afrika - wesentliche Einflüsse vermittelte. In dieser Hinsicht wäre es durchaus einmal lohnenswert, einen Fernando Ortiz mit dem Franzosen Michel Leiris zu vergleichen. Wir sehen gerade in diesem Bereich auch die Anknüpfungspunkte zur Studentenbewegung im ersten Drittel des 20. Jahrhunderts, die ähnliche Forderungen - insbesondere die grundlegende Veränderung des auf eine gesellschaftliche Elite zielenden Bildungssystems - auf ihre Fahnen geschrieben hatten.

Siebtens ist für die historischen Avantgarden in Lateinamerika der kulturelle Pol internationaler Massenkommunikation von wachsender Bedeutung, da er nicht allein die Problematik der überregionalen und internationalen Zeitschriften, sondern vor allem auch eine breite Palette an volkskulturellen Praktiken und Ausdrucksformen betrifft, die nun in diesen kulturellen Pol integriert und international distribuiert werden. Als Beispiele für eine derartige Internationali- 
sierung mögen die verschiedenen Spielarten des argentinischen Tango oder des kubanischen Son gelten, welche über Frankreich und die USA weltweit verbreitet wurden. Daran schließen sich in Lateinamerika wiederum literarische Praktiken von Liedtexten bis hin zu großen Erzählformen an.

Die Internationalisierung lateinamerikanischer Kultur und Literatur ist damit ein wesentliches Faktum der entsprechenden historischen Avantgarden, blieb selbstverständlich aber nicht auf diese beschränkt, wie wir am Beispiel der uruguayischen Dichterin Juana de Ibarbourou erkennen werden. Doch zeigt sich die große Relevanz dieses kulturellen Pols erstmals massiv bei den Generationen der während der zwanziger und dreißiger Jahre schreibenden Schriftsteller, also zur Blütezeit der historischen Avantgarden. Letztlich zielen auch diese Entwicklungen auf eine größere und intensivere Partizipation und damit auf eine stärkere Konvivenz der Kulturen, die als Zielstellung eindeutig mit den historischen Avantgarden in Lateinamerika zu verbinden ist.

Achtens und vorerst letztens scheint sich innerhalb der avantgardistischen literarischen Schreibformen vor allem im Bereich der Lyrik eine eigene Traditionslinie schreibender Frauen herausgebildet zu haben, die sich - zumindest gemäß der Argumentation eines José Carlos Mariátegui - deutlich von Formen der von Männern geschriebenen Lyrik emanzipiert. Beispiele hierfür sind unter anderem Alfonsina Storni, Juana de Ibarbourou und Gabriela Mistral, die als erste lateinamerikanische Autorin und lange vor allen männlichen Schriftstellern ihres Kontinents 1945 mit dem Nobelpreis für Literatur ausgezeichnet wurde.

Wir werden uns im übernächsten Schritt mit diesen Dichterinnen näher auseinandersetzen, da die Entfaltung ihres Schreibens in gewisser Weise quer zur Entwicklung der historischen Avantgarden verlief. Dass es gerade den Lyrikerinnen (und weniger ihren männlichen Kollegen) beschieden war, auf eine breite nationale wie internationale Aufnahme zu stoßen und internationale Preise bis hinauf zum Literaturnobelpreis einzuheimsen, ist ein interessantes Paradoxon der hispanoamerikanischen Literaturgeschichte, das zugleich den dann sinkenden Stern der historischen Avantgarden signalisierte. Doch werden wir zugleich auch sehen, dass es aus den historischen Avantgarden viele Wege nicht allein in die Neo-Avantgarden, sondern vor allem auch in Formen postmoderner Ästhetiken gibt. Nun aber soll unsere Vorlesung zunächst einmal große Figuren der lateinamerikanischen Avantgarden präsentieren und deren Werke anschaulich nachvollziehbar machen. 